\title{
Convergence Theorems for Operators Sequences on Functionals of Discrete-Time Normal Martingales
}

\author{
Jinshu Chen \\ School of Science, Lanzhou University of Technology, Lanzhou, Gansu 730050, China \\ Correspondence should be addressed to Jinshu Chen; emily-chen@126.com
}

Received 6 November 2017; Revised 26 December 2017; Accepted 3 January 2018; Published 4 February 2018

Academic Editor: Alberto Fiorenza

Copyright (C) 2018 Jinshu Chen. This is an open access article distributed under the Creative Commons Attribution License, which permits unrestricted use, distribution, and reproduction in any medium, provided the original work is properly cited.

\begin{abstract}
We aim to investigate the convergence of operators sequences acting on functionals of discrete-time normal martingales $M$. We first apply the 2D-Fock transform for operators from the testing functional space $\delta(M)$ to the generalized functional space $\mathcal{S}^{*}(M)$ and obtain a necessary and sufficient condition for such operators sequences to be strongly convergent. We then discuss the integration of these operator-valued functions. Finally, we apply the results obtained here and establish the existence and uniqueness of solution to quantum stochastic differential equations in terms of operators acting on functionals of discrete-time normal martingales $M$. And also we prove the continuity and continuous dependence on initial values of the solution.
\end{abstract}

\section{Introduction}

White noise analysis created by Hida [1] is essentially a branch of infinite-dimensional calculus on generalized functionals of Brownian motion, which connected with the applications to the study of random processes and stochastic differential equations. Ito's theory is based on the notion of the Wiener measure in the space of continuous functions, interpreted as the trajectories of Brownian motion. And considering the space of slowly growing Schwartz distributions as the space of trajectories of white noise, Hida has constructed a new theory of white noise functionals [2-4], which proved to be useful in the studying of stochastic differential equations and was further developed in $[5,6]$ and other papers. On the other hand, many problems in mathematical physics and financial mathematics lead to the necessity of studying stochastic differential equations with operator coefficients in infinitedimensional spaces. In [7], the authors generalize the classical Ito theory to this case.

In 1998, Ito introduced his analysis of generalized Poisson functionals in paper [8], which can be viewed as a theory of infinite-dimensional calculus on generalized functionals of Poisson martingale. As we know, both Brownian motion and Poisson martingale are continuous-time normal martingales. And other continuous-time processes theories of white noise analysis appeared in [9-12].
Discrete-time normal martingales [13] also play an important role in many theoretical and applied fields. For example, the classical random walk is just such a discretetime normal martingale $[14,15]$. It would then be interesting to develop a theory of infinite-dimensional calculus on generalized functionals of discrete-time normal martingales.

Let $M=\left(M_{n}\right)_{n \in \mathbb{N}}$ be a discrete-time normal martingale satisfying some mild conditions. In paper [16], the authors constructed the testing functional space $\mathcal{S}(M)$ and generalized functional space $\mathcal{S}^{*}(M)$ of $M$ by using a specific orthonormal basis for square integrable functionals of $M$ and also characterized these functionals via a transform acting on them.

It is well known that operators on functional spaces play a fundamental role in quantum mechanics. In paper [17], the authors introduce a transform, called 2D-Fock transform, for operators from the testing functional space $\mathcal{S}(M)$ to the generalized functional space $\mathcal{S}^{*}(M)$ of $M$ and characterize continuous linear operators from $\delta(M)$ to $\delta^{*}(M)$. In this paper, we apply the $2 \mathrm{D}$-Fock transform to investigate the convergence of operators sequences acting on functionals of discrete-time normal martingales $M$. We obtain a necessary and sufficient condition for such sequence to be strongly convergent. And we give a criterion for checking whether such operator-valued functions are Bochner-integrable. 
Finally, as application of the results obtained here, we investigate the following nonlinear quantum stochastic differential equation:

$$
\begin{aligned}
\frac{d T(t)}{d t} & =X(t, T(t))+Y(t, T(t)) * N(t), \quad t \in \mathbb{R}_{+} \\
T(0) & =J,
\end{aligned}
$$

where $X, Y: \mathbb{R} \times \mathscr{L}\left[\mathcal{S}(M), \mathcal{S}^{*}(M)\right] \rightarrow \mathscr{L}\left[\mathcal{S}(M), \mathcal{S}^{*}(M)\right]$ are two maps, $J \in \mathscr{L}\left[\mathcal{S}(M), \mathcal{S}^{*}(M)\right]$ is the initial value, and * stands for the convolution of operators. $(N(t))_{t \in \mathbb{R}_{+}}$is a given $\mathscr{L}\left[\mathcal{S}(M), \mathcal{S}^{*}(M)\right]$-valued quantum stochastic process which plays the role of quantum noises, and the solution $(T(t))_{t \in \mathbb{R}_{+}}$ will be a $\mathscr{L}\left[\mathcal{S}(M), \mathcal{S}^{*}(M)\right]$-valued quantum stochastic process.

Equation (1) can describe the evolution of a quantum system at a level of operators in the presence of quantum noise. We will establish the existence and uniqueness of solution to equation (1). And also we prove the continuity and continuous dependence on initial values of the solution.

The paper is organized as follows. In Section 2, we briefly recall the construction and characterization of continuous linear operators from $\mathcal{S}(M)$ to $\mathcal{S}^{*}(M)$, which were made in recent paper [17]. In Section 3, we apply the 2D-Fock transform to investigate the convergence of operators sequences acting on functionals of discrete-time normal martingales $M$. We first obtain a necessary and sufficient condition for such sequence to be strongly convergent. And then we give a criterion for checking whether such operator-valued functions are Bochner-integrable. In Section 4, we apply the results obtained here and establish the existence and uniqueness of solution to quantum stochastic differential equations (1) and prove the continuity and continuous dependence on initial values of the solution.

Notation and Conventions. Throughout the paper, $\mathbb{N}$ designates the set of all nonnegative integers and $\Gamma$ denotes the finite power set of $\mathbb{N}$; namely,

$$
\Gamma=\{\sigma \mid \sigma \subset \mathbb{N}, \#(\sigma)<\infty\},
$$

where $\#(\sigma)$ means the cardinality of $\sigma$ as a set. In addition, we always assume that $(\Omega, \mathscr{F}, P)$ is a given probability space with $\mathbb{E}$ denoting the expectation with respect to $P$. We denote by $L^{2}(\Omega, \mathscr{F}, P)$ the usual Hilbert space of square integrable complex-valued functions on $(\Omega, \mathscr{F}, P)$ and use $\langle\cdot, \cdot\rangle$ and $\|\cdot\|$ to mean its inner product and norm, respectively. By convention, $\langle\cdot, \cdot\rangle$ is conjugate-linear in its first argument and linear in its second argument.

\section{Generalized Functionals of Discrete-Time Normal Martingales}

Definition 1. A stochastic process $M=\left(M_{n}\right)_{n \in \mathbb{N}}$ on $(\Omega, \mathscr{F}, P)$ is called a discrete-time normal martingale if it is square integrable and satisfies

(i) $\mathbb{E}\left[M_{0} \mid \mathscr{F}_{-1}\right]=0$ and $\mathbb{E}\left[M_{n} \mid \mathscr{F}_{n-1}\right]=M_{n-1}$ for $n \geq 1$; (ii) $\mathbb{E}\left[M_{0}^{2} \mid \mathscr{F}_{-1}\right]=1$ and $\mathbb{E}\left[M_{n}^{2} \mid \mathscr{F}_{n-1}\right]=M_{n-1}^{2}+1$ for $n \geq 1$,

where $\mathscr{F}_{-1}=\{\emptyset, \Omega\}, \mathscr{F}_{n}=\sigma\left(M_{k} ; 0 \leq k \leq n\right)$ for $n \in \mathbb{N}$, and $\mathbb{E}\left[\cdot \mid \mathscr{F}_{k}\right]$ means the conditional expectation.

Let $M=\left(M_{n}\right)_{n \in \mathbb{N}}$ be a discrete-time normal martingale on $(\Omega, \mathscr{F}, P)$. Then one can construct from $M$ a process $Z=$ $\left(Z_{n}\right)_{n \in \mathbb{N}}$ as

$$
\begin{aligned}
& Z_{0}=M_{0}, \\
& Z_{n}=M_{n}-M_{n-1}, \quad n \geq 1 .
\end{aligned}
$$

It can be verified that $Z$ admits the following properties:

$$
\begin{aligned}
\mathbb{E}\left[Z_{n} \mid \mathscr{F}_{n-1}\right] & =0, \\
\mathbb{E}\left[Z_{n}^{2} \mid \mathscr{F}_{n-1}\right] & =1, \\
n & \in \mathbb{N} .
\end{aligned}
$$

Thus $Z$ can be viewed as a discrete-time noise, which we call the discrete-time normal noise associated with $M$.

Lemma 2 (see $[18,19]$ ). Let $Z=\left(Z_{n}\right)_{n \in \mathbb{N}}$ be the discretetime normal noise associated with $M$. Define $Z_{\emptyset}=1$, where $\emptyset$ denotes the empty set and

$$
Z_{\sigma}=\prod_{i \in \sigma} Z_{i}, \quad \sigma \in \Gamma, \sigma \neq \emptyset .
$$

Then $\left\{Z_{\sigma} \mid \sigma \in \Gamma\right\}$ forms a countable orthonormal system in $L^{2}(\Omega, \mathscr{F}, P)$.

Let $\mathscr{F}_{\infty}=\sigma\left(M_{n} ; n \in \mathbb{N}\right)$; the $\sigma$-field over $\Omega$ is generated by $M$. In the literature, $\mathscr{F}_{\infty}$-measurable functions on $\Omega$ are also known as functionals of $M$. Thus elements of $L^{2}(\Omega$, $\mathscr{F}_{\infty}, P$ ) can be called square integrable functionals of $M$.

Definition 3. The discrete-time normal martingale $M$ is said to have the chaotic representation property if the system $\left\{Z_{\sigma} \mid\right.$ $\sigma \in \Gamma\}$ defined by (5) is total in $L^{2}\left(\Omega, \mathscr{F}_{\infty}, P\right)$.

So, if the discrete-time normal martingale $M$ has the chaotic representation property, then the system $\left\{Z_{\sigma} \mid \sigma \in\right.$ $\Gamma$ \} defined by (5) is actually an orthonormal basis for $L^{2}(\Omega$, $\left.\mathscr{F}_{\infty}, P\right)$, which is a closed subspace of $L^{2}(\Omega, \mathscr{F}, P)$ as is known.

And, for brevity, we use $L^{2}(M)$ to mean the space of square integrable functionals of $M$; namely,

$$
L^{2}(M)=L^{2}\left(\Omega, \mathscr{F}_{\infty}, P\right),
$$

which shares the same inner product and norm with $L^{2}(\Omega, \mathscr{F}, P)$, namely, $\langle\cdot, \cdot\rangle$ and $\|\cdot\|$. We note that $\left\{Z_{\sigma} \mid \sigma \in \Gamma\right\}$ forms a countable orthonormal basis for $L^{2}(M)$.

Lemma 4 (see [20]). Let $\sigma \mapsto \lambda_{\sigma}$ be the $\mathbb{N}$-valued function on $\Gamma$ given by

$$
\lambda_{\sigma}= \begin{cases}\prod_{k \in \sigma}(k+1), & \sigma \neq \emptyset, \sigma \in \Gamma \\ 1, & \sigma=\emptyset, \sigma \in \Gamma .\end{cases}
$$


Then, for $p>1$, the positive term series $\sum_{\sigma \in \Gamma} \lambda_{\sigma}^{-p}$ converges and moreover

$$
\sum_{\sigma \in \Gamma} \lambda_{\sigma}^{-p} \leq \exp \left[\sum_{k=1}^{\infty} k^{-p}\right]<\infty .
$$

Using the $\mathbb{N}$-valued function defined by (7), we can construct a chain of Hilbert spaces consisting of functionals of $M$ as follows. For $p \geq 0$, we define a norm $\|\cdot\|_{p}$ on $L^{2}(M)$ through

$$
\|\xi\|_{p}^{2}=\sum_{\sigma \in \Gamma} \lambda_{\sigma}^{2 p}\left|\left\langle Z_{\sigma}, \xi\right\rangle\right|^{2}, \quad \xi \in L^{2}(M)
$$

and put

$$
\delta_{p}(M)=\left\{\xi \in L^{2}(M) \mid\|\xi\|_{p}<\infty\right\} .
$$

It is not hard to check that $\|\cdot\|_{p}$ is a Hilbert norm and $\delta_{p}(M)$ becomes a Hilbert space with $\|\cdot\|_{p}$.

Lemma 5 (see [16]). For $p \geq 0$, one has $\left\{Z_{\sigma} \mid \sigma \in \Gamma\right\} \subset$ $\mathcal{S}_{p}(M)$ and moreover the system $\left\{\lambda_{\sigma}^{-p} Z_{\sigma} \mid \sigma \in \Gamma\right\}$ forms an orthonormal basis for $\mathcal{S}_{p}(M)$.

It is easy to see that $\lambda_{\sigma} \geq 1$ for all $\sigma \in \Gamma$. This implies that $\|\cdot\|_{p} \leq\|\cdot\|_{q}$ and $\delta_{q}(M) \subset \delta_{p}(M)$ whenever $0 \leq p \leq q$. Thus we actually get a chain of Hilbert spaces of functionals of $M$ :

$$
\begin{aligned}
\cdots & \subset \mathcal{S}_{p+1}(M) \subset \mathcal{S}_{p}(M) \subset \cdots \subset \mathcal{S}_{1}(M) \subset \mathcal{S}_{0}(M) \\
& =L^{2}(M) .
\end{aligned}
$$

We now put

$$
\mathcal{S}(M)=\bigcap_{p=0}^{\infty} \mathcal{S}_{p}(M)
$$

and endow it with the topology generated by the norm sequence $\left\{\|\cdot\|_{p}\right\}_{p \geq 0}$. Note that, for each $p \geq 0, \delta_{p}(M)$ is just the completion of $\mathcal{S}(M)$ with respect to $\|\cdot\|_{p}$. Thus $\mathcal{S}(M)$ is a countably-Hilbert space [21]. The next lemma, however, shows that $\mathcal{S}(M)$ even has a much better property.

Lemma 6 (see [16]). The space $\mathcal{S}(M)$ is a nuclear space; namely, for any $p \geq 0$, there exists $q>p$ such that the inclusion mapping $i_{p q}: \mathcal{S}_{q}(M) \rightarrow \mathcal{S}_{p}(M)$ defined by $i_{p q}(\xi)=\xi$ is a Hilbert-Schmidt operator.

For $p \geq 0$, we denote by $\mathcal{S}_{p}^{*}(M)$ the dual of $\mathcal{\delta}_{p}(M)$ and by $\|\cdot\|_{-p}$ the norm of $\mathcal{S}_{p}^{*}(M)$. Then $\mathcal{S}_{p}^{*}(M) \subset \mathcal{S}_{q}^{*}(M)$ and $\|\cdot\|_{-p} \geq$ $\|\cdot\|_{-q}$ whenever $0 \leq p \leq q$. The lemma below is then an immediate consequence of the general theory of countablyHilbert spaces (see [21]).

Lemma 7 (see [16]). Let $\mathcal{S}^{*}(M)$ be the dual of $\mathcal{S}(M)$ and endow it with the strong topology. Then

$$
\mathcal{S}^{*}(M)=\bigcup_{p=0}^{\infty} \mathcal{S}_{p}^{*}(M)
$$

and moreover the inductive limit topology on $\mathcal{S}^{*}(M)$ given by space sequence $\left\{\mathcal{S}_{p}^{*}(M)\right\}_{p \geq 0}$ coincides with the strong topology.
We mention that, by identifying $L^{2}(M)$ with its dual, one comes to a Gel'fand triple,

$$
\mathcal{S}(M) \subset L^{2}(M) \subset \mathcal{S}^{*}(M),
$$

which we refer to as the Gel'fand triple associated with $M$.

Definition 8 (see [16]). Elements of $\mathcal{S}^{*}(M)$ are called generalized functionals of $M$, while elements of $\mathcal{S}(M)$ are called testing functionals of $M$.

Throughout this paper, we denote by $\mathscr{L}$ the set of all continuous linear operators from $\mathcal{S}(M)$ to $\mathcal{S}^{*}(M)$; that is, $\mathscr{L}=\mathscr{L}\left[\mathcal{S}(M), \mathcal{S}^{*}(M)\right]$.

Definition 9 (see [17]). For an operator $T \in \mathscr{L}$, its 2D-Fock transform is the function $\widehat{T}$ on $\Gamma \times \Gamma$ given by

$$
\widehat{T}(\sigma, \tau)=\left\langle\left\langle T\left(Z_{\sigma}\right), Z_{\tau}\right\rangle\right\rangle, \quad(\sigma, \tau) \in \Gamma \times \Gamma,
$$

where $\langle\langle\cdot, \cdot\rangle\rangle$ is the canonical bilinear form on $\mathcal{S}^{*}(M) \times$ $\mathcal{S}(M)$.

Much like generalized functionals of $M$, continuous linear operators in $\mathscr{L}$ are also completely determined by their 2D-Fock transforms.

Lemma 10 (see [17]). Let $T_{1}, T_{2}: \mathcal{S}(M) \rightarrow \mathcal{S}^{*}(M)$ be continuous linear operators. Then $T_{1}=T_{2}$ if and only if $\widehat{T_{1}}=$ $\widehat{T_{2}}$.

The following lemma is known as the characterization theorem of operators in $\mathscr{L}$ through their 2D-Fock transforms.

Lemma 11 (see [17]). Let $G$ be a function on $\Gamma \times \Gamma$. Then $G$ is the $2 D$-Fock transform of an element $T$ in $\mathscr{L}$ if and only if it satisfies

$$
|G(\sigma, \tau)| \leq C \lambda_{\sigma}^{p} \lambda_{\tau}^{p}, \quad(\sigma, \tau) \in \Gamma \times \Gamma
$$

for some constants $C \geq 0$ and $p \geq 0$. In that case, for $q>$ $p+1 / 2$, one has

$$
\|T\|_{q} \leq C \sum_{\sigma \in \Gamma} \lambda_{\sigma}^{-2(q-p)}
$$

and in particular $T$ takes values in $\mathcal{S}_{q}^{*}(M)$, where

$$
\|T\|_{p} \equiv \sup _{\xi \neq 0, \xi \in \mathcal{S}(M)} \frac{\|T(\xi)\|_{-p}}{\|\xi\|_{p}},
$$

where $p \geq 0$.

For two operators $T_{1}, T_{2} \in \mathscr{L}$, their usual product $T_{1} T_{2}$ may not make sense. However, one can introduce a product of other type for them.

Definition 12 (see [17]). Let $T_{1}, T_{2} \in \mathscr{L}$; then their convolution $T_{1} * T_{2}$ is defined as

$$
\widehat{T_{1} * T_{2}}(\sigma, \tau)=\widehat{T}_{1}(\sigma, \tau) \widehat{T}_{2}(\sigma, \tau), \quad(\sigma, \tau) \in \Gamma \times \Gamma .
$$

It can be verified that $(\mathscr{L}, *)$ forms a commutative algebra with an involution and a unit. 


\section{Convergence Theorems for Operators in $\mathscr{L}$}

Let $M=\left(M_{n}\right)_{n \in \mathbb{N}}$ be the same discrete-time normal martingale as described in Section 2. In the present section, we apply the 2D-Fock transform to establish convergence theorems for operators in $\mathscr{L}$. Furthermore, we discuss the integration of these operator-valued functions.

Definition 13. A sequence $\left(T_{n}\right)_{n \geq 1} \subset \mathscr{L}$ is called strongly convergent to $T \in \mathscr{L}$, if, for any $\xi \in \mathcal{S}(M)$, one has $T_{n}(\xi) \rightarrow$ $T(\xi)$ (in the strong topology of $\mathcal{S}^{*}(M)$ ).

The following theorem offers a necessary condition for operators sequences in $\mathscr{L}$ to be strongly convergent.

Theorem 14. Let $\left(T_{n}\right)_{n \geq 1}$ be an operator sequence in $\mathscr{L}$, and let $T$ be operator in $\mathscr{L}$. Then the sequence $\left(T_{n}\right)_{n \geq 1}$ converges strongly to $T$ if and only if

(1) $\widehat{T_{n}}(\sigma, \tau) \rightarrow \widehat{T}(\sigma, \tau)$ for all $\sigma, \tau \in \Gamma$;

(2) there exist constants $C \geq 0$ and $p \geq 0$ such that

$$
\sup _{n \geq 1}\left|\widehat{T_{n}}(\sigma, \tau)\right| \leq C \lambda_{\sigma}^{p} \lambda_{\tau}^{p}, \quad(\sigma, \tau) \in \Gamma \times \Gamma
$$

Proof. For the "only if" part, let $\left(T_{n}\right)_{n \geq 1}$ converge strongly to $T$; then it is easy to see that

$$
\begin{aligned}
\widehat{T_{n}}(\sigma, \tau) & =\left\langle\left\langle T_{n}\left(Z_{\sigma}\right), Z_{\tau}\right\rangle\right\rangle \longrightarrow\left\langle\left\langle T\left(Z_{\sigma}\right), Z_{\tau}\right\rangle\right\rangle \\
& =\widehat{T}(\sigma, \tau), \quad \sigma, \tau \in \Gamma .
\end{aligned}
$$

On the other hand, by Lemma 11, we know that, for each $n$, there exist $C_{n} \geq 0$ and $p \geq 0$ such that

$$
\left|\widehat{T}_{n}(\sigma, \tau)\right| \leq C_{n} \lambda_{\sigma}^{p} \lambda_{\tau}^{p}, \quad(\sigma, \tau) \in \Gamma \times \Gamma
$$

Let $C=\sup _{n \geq 1} C_{n}$, according to the principle of uniform boundedness, $C<\infty$, which implies that

$$
\sup _{n \geq 1}\left|\widehat{T_{n}}(\sigma, \tau)\right| \leq C \lambda_{\sigma}^{p} \lambda_{\tau}^{p}, \quad(\sigma, \tau) \in \Gamma \times \Gamma .
$$

For the "if" part, let $\left(T_{n}\right)_{n \geq 1}$ satisfy conditions (1) and (2); by taking $q>p+1 / 2$ and using Lemma 11, we get

$$
\sup _{n \geq 1}\left\|T_{n}(\xi)\right\|_{-q} \leq C \sum_{\sigma \in \Gamma} \lambda_{\sigma}^{-2(q-p)}\|\xi\|_{q}, \quad \xi \in \mathcal{S}(M)
$$

which means that $\left(T_{n}\right)_{n \geq 1}$ is an operator sequence from $\mathcal{S}_{q}(M)$ to $\mathcal{S}_{q}^{*}(M)$ and it is uniformly bounded. On the other hand, $\left\{Z_{\sigma} \mid \sigma \in \Gamma\right\}$ is total in $\mathcal{S}_{q}(M)$, and condition (1) equivalent to $\left\langle\left\langle T_{n}\left(Z_{\sigma}\right), Z_{\tau}\right\rangle\right\rangle$ converges in $\mathbb{C}$ for any $\sigma, \tau \in \Gamma$, so there exists a linear operator form $\mathcal{S}_{q}(M)$ to $\mathcal{S}_{q}^{*}(M)$ such that, for any $\xi, \eta \in \mathcal{S}_{q}(M)$, we have

$$
\left\langle\left\langle T_{n}(\xi), \eta\right\rangle\right\rangle \longrightarrow\langle\langle T(\xi), \eta\rangle\rangle \quad(n \longrightarrow \infty) ;
$$

thus $T$ is a linear operator form $\mathcal{S}(M)$ to $\mathcal{S}^{*}(M)$, and, for any $\xi \in \mathcal{S}(M), T_{n}(\xi)$ converges weakly to $T(\xi)$ in $\mathcal{S}^{*}(M)$. By Proposition 3.1 in paper [16], $T_{n}(\xi)$ converges strongly to $T(\xi)$ in $\mathcal{S}^{*}(M)$. So $\left(T_{n}\right)_{n \geq 1}$ converges strongly to $T$.
In the following, we discuss the integration of $\mathscr{L}$-valued mapping.

Definition 15. Let $(E, \mathscr{E}, \nu)$ be a measure space and let $T(\cdot)$ : $E \rightarrow \mathscr{L}$ be a $\mathscr{L}$-valued function; then $T(\cdot)$ is said to be strongly Bochner-integrable with respect to $v$ if generalized functional valued function $T(\cdot) \xi: E \rightarrow \mathcal{S}^{*}(M)$ is Bochnerintegrable with respect to $\nu$ for any $\xi \in \mathcal{S}(M)$. In that case, a linear operator from $\mathcal{S}(M)$ to $\mathcal{S}^{*}(M)$,

$$
\xi \longrightarrow \int_{E} T(\omega) \xi d \nu(\omega)
$$

is defined. We called it the Bochner integral of $T(\cdot)$ with respect to $\nu$ and it is denoted by $\int_{E} T(\omega) d \nu(\omega)$.

We note that the strong Bochner integral $\int_{E} T(\omega) d \nu(\omega)$ may not be an operator in $\mathscr{L}$. The next theorem, however, provides sufficient conditions for $T(\cdot)$ to be strongly Bochnerintegrable and its strong Bochner integral to be an operator in $\mathscr{L}$.

Theorem 16. Let $T(\cdot): E \rightarrow \mathscr{L}$ be a $\mathscr{L}$-valued mapping satisfying the following conditions:

(1) For any $\sigma, \tau \in \Gamma$, the function $\widehat{T(\cdot)}(\sigma, \tau): E \rightarrow \mathbb{C}$ is measurable.

(2) There exist $p \geq 0$ and a nonnegative function $C(\omega) \epsilon$ $L^{1}(E, v)$, such that, for $v$-a.e., $\omega \in E$, it holds that

$$
|\widehat{T(\omega)}(\sigma, \tau)| \leq C(\omega) \lambda_{\sigma}^{p} \lambda_{\tau}^{p} \quad \sigma, \tau \in \Gamma
$$

Then $T(\cdot)$ is strongly Bochner-integrable with respect to $v$ and its strong Bochner integral satisfies the following norm inequality:

$$
\left\|\int_{E} T(\omega) d \nu(\omega)\right\|_{q} \leq \sum_{\sigma \in \Gamma} \lambda_{\sigma}^{-2(q-p)} \int_{E} C(\omega) d \nu(\omega),
$$

where $q>p+1 / 2$. In particular, $\int_{E} T(\omega) d \nu(\omega) \in \mathscr{L}$.

Proof. We take $q>p+1 / 2$. Then, by Lemma 11, for $\nu$-a.e. $\omega \in E$, we have

$$
\|T(\omega) \xi\|_{-q} \leq C(\omega) \sum_{\sigma \in \Gamma} \lambda_{\sigma}^{-2(q-p)}\|\xi\|_{q}, \quad \sigma \in \Gamma
$$

which means that $T(\cdot)$ is an operator from $\mathcal{S}_{q}(M)$ to $\mathcal{S}_{q}^{*}(M)$. On the other hand, according to (1), for any $\xi, \eta \in \mathcal{S}_{q}^{*}(M)$, $\langle\langle T(\omega) \xi, \eta\rangle\rangle: E \rightarrow \mathbb{C}$ is measurable. And $\mathcal{S}_{q}^{*}(M)$ is a separable space, so $T(\omega) \xi: E \rightarrow \mathcal{S}_{q}^{*}(M)$ is strongly measurable for any $\xi \in \mathcal{S}_{q}^{*}(M)$, where, together with (27), for any $\xi \in$ $\mathcal{S}_{q}^{*}(M)$, the map

$$
T(\cdot) \xi: E \longrightarrow \mathcal{S}_{q}^{*}(M)
$$

is Bochner-integrable with respect to $\nu$. Thus, $T(\cdot): E \rightarrow \mathscr{L}$ is strongly Bochner-integrable with respect to $\nu$. Finally, (28) easily follows from (29) and we find $\int_{E} T(\omega) d \nu(\omega) \in \mathscr{L}$. 
Definition 17. Let $0 \leq L<+\infty$; the map $T(\cdot):[0, L] \rightarrow \mathscr{L}$ is said to be sequentially continuous; if $\left(t_{n}\right)_{n \geq 1}$ is a sequence in $[0, L]$ and $\left(t_{n}\right)_{n \geq 1}$ converges to $t_{0} \in[0, L]$, we have that $T\left(t_{n}\right)$ converges to $T\left(t_{0}\right)$.

Definition 17 is the notion of sequentially continuous, because $\mathscr{L}$-valued map $T(\cdot)$ is defined on interval, so it is equivalent to continuous. The next theorem offers a necessary and sufficient condition for $\mathscr{L}$-valued functions to be continuous.

Theorem 18. Let $0 \leq L<+\infty ; T(\cdot):[0, L] \rightarrow \mathscr{L}$ is a $\mathscr{L}$ valued function; then $T(\cdot)$ is continuous if and only if

(1) $\widehat{T(\cdot)}(\sigma, \tau):[0, L] \rightarrow \mathbb{C}$ is continuous for any $\sigma, \tau \in \Gamma$;

(2) there exist $p \geq 0$ and $C \geq 0$ such that

$$
|\widehat{T(\cdot)}(\sigma, \tau)| \leq C \lambda_{\sigma}^{p} \lambda_{\tau}^{p}
$$

for each $\sigma, \tau \in \Gamma$ and $t \in[0, L]$.

Proof. We just prove the "if" part. Let $t_{0} \in[0, L]$ and $\left(t_{n}\right)_{n \geq 1} \subset$ $[0, L]$ converges to $t_{0}$; then the sequence $\left(T\left(t_{n}\right)\right)_{n \geq 1} \in \mathscr{L}$ and satisfies the following conditions:

(1) For every $\sigma \in \Gamma, \widehat{T\left(t_{n}\right)}(\sigma, \tau) \rightarrow \widehat{T\left(t_{0}\right)}(\sigma, \tau)$.

(2) There exist $p \geq 0$ and $C \geq 0$, such that

$$
\left|\widehat{T\left(t_{n}\right)}(\sigma, \tau)\right| \leq C \lambda_{\sigma}^{p} \lambda_{\tau}^{p}, \quad \sigma \in \Gamma
$$

which implies that the operator sequence $\left(T\left(t_{n}\right)\right)_{n \geq 1}$ satisfies the conditions in Theorem 14; thus, there exists $S \in \mathscr{L}$ such that $T\left(t_{n}\right) \rightarrow S$, and

$$
\widehat{T\left(t_{0}\right)}(\sigma, \tau)=\lim _{n \rightarrow \infty} \widehat{T\left(t_{n}\right)}(\sigma, \tau)=\widehat{S}(\sigma, \tau) .
$$

Then $T\left(t_{0}\right)=S$, which implies that

$$
T\left(t_{n}\right) \longrightarrow T\left(t_{0}\right)
$$

and $T(\cdot)$ is continuous.

\section{Application to Quantum SDEs}

In the present section, we show applications of our main results in Section 3. We establish the existence and uniqueness of solution to (1). Equation (1) can describe quantum stochastic evolutions in the presence of quantum noise. As usual, by a solution of (1) we mean a map $T: \mathbb{R}_{+} \rightarrow \mathscr{L}$ which satisfies the following integral equation:

$$
\begin{aligned}
T(t)= & J+\int_{0}^{t} X(s, T(s)) d s \\
& +\int_{0}^{t} Y(s, T(s)) * N(s) d s, \quad t \in \mathbb{R}_{+},
\end{aligned}
$$

where the integral is the Bochner integral of operator function in $\mathscr{L}$.
Definition 19. Let $\Theta: \mathbb{R}_{+} \times \mathscr{L} \rightarrow \mathscr{L}$ be a map. $\Theta$ is said to satisfy a locally uniform Lipschitz condition and a locally uniform linear growth condition, if, for any $L \geq 0$, there exists a constant $\rho \geq 0$, such that, for any $\sigma, \tau \in \Gamma$ and $A, B \in \mathscr{L}$, one has

$$
\begin{aligned}
& |\widehat{\Theta(t, A)}(\sigma, \tau)-\widehat{\Theta(t, B)}(\sigma, \tau)| \\
& \quad \leq \rho|\widehat{A}(\sigma, \tau)-\widehat{B}(\sigma, \tau)|, \quad t \in[0, L], \\
& |\widehat{\Theta(t, A)}(\sigma, \tau)| \leq \rho(1+|\widehat{A}(\sigma, \tau)|), \quad t \in[0, L] .
\end{aligned}
$$

Theorem 20. Let $X, Y: \mathbb{R}_{+} \times \mathscr{L} \rightarrow \mathscr{L}$ be continuous and satisfy a locally uniform Lipschitz condition and locally uniform linear growth condition, and let the noise process $(N(t))_{t \in \mathbb{R}_{+}}$satisfy the following:

(1) $N: \mathbb{R}_{+} \rightarrow \mathscr{L}$ is continuous.

(2) For any $L \geq 0$, there exists a constant $K \geq 1$, such that, for any $\sigma, \tau \in \Gamma$,

$$
|\widehat{N(t)}(\sigma, \tau)| \leq K, \quad t \in[0, L] .
$$

Then, for any $J \in \mathscr{L}$, there exists a unique continuous map $T: \mathbb{R}_{+} \rightarrow \mathscr{L}$, solving (1).

Proof. We just prove that, for any $L \geq 0$, the integral equation (35) has a unique continuous solution on $[0, L]$.

First, using iteration, we will prove that, for each $n \geq 1$, there exists a sequence $T_{n}:[0, L] \rightarrow \mathscr{L}$, which has the following properties:

(1) For any $\sigma, \tau \in \Gamma$, the function $\widehat{T_{n}(\cdot)}(\sigma, \tau):[0, L] \rightarrow \mathbb{C}$ is continuous.

(2) For any $\sigma, \tau \in \Gamma$ and $t \in[0, L]$, there exists a constant $\alpha>0$, such that

$$
\left|\widehat{T_{n}(t)}(\sigma, \tau)\right| \leq \alpha \lambda_{\sigma}^{p} \lambda_{\tau}^{p}+\left(1+\alpha \lambda_{\sigma}^{p} \lambda_{\tau}^{p}\right) \sum_{i=1}^{n} \frac{\rho^{i} t^{i} K^{i}}{i !} .
$$

(3)

$$
\begin{aligned}
T_{n}(t)= & J+\int_{0}^{t} X\left(s, T_{n-1}(s)\right) d s+\int_{0}^{t} Y\left(s, T_{n-1}(s)\right) \\
& * N(s) d s,
\end{aligned}
$$

where $T_{0}(s)=J$.

In fact, from the assumptions and Theorem 16 , for any $t \epsilon$ $[0, L]$, the integral $\int_{0}^{t} X(s, J) d s$ and $\int_{0}^{t} Y(s, J) * N(s) d s$ exist and belong to $\mathscr{L}$. Now we define $T_{1}:[0, L] \rightarrow \mathscr{L}$ as follows:

$$
\begin{array}{r}
T_{1}(t)=J+\int_{0}^{t} X(s, J) d s+\int_{0}^{t} Y(s, J) * N(s) d s, \\
t \in[0, L] .
\end{array}
$$

It is easy to see that, for any $\sigma, \tau \in \Gamma$, the function $\widehat{T_{1}(\cdot)}(\sigma, \tau)$ : $[0, L] \rightarrow \mathbb{C}$ is continuous. On the other hand, from the 
assumptions, there exist constants $p \geq 0, \alpha>0$, and $\rho>0$, such that

$$
\begin{aligned}
& \widehat{T_{1}(t)}(\sigma, \tau) \\
& \leq|\widehat{J}(\sigma, \tau)|+\int_{0}^{t}|\widehat{X(s, J)}(\sigma, \tau)| d s \\
& \quad+\int_{0}^{t}|\widehat{Y(s, J)}(\sigma, \tau)||\widehat{N(s)}(\sigma, \tau)| d s \\
& \leq \alpha \lambda_{\sigma}^{p} \lambda_{\tau}^{p} \\
&+K \int_{0}^{t}(|\widehat{X(s, J)}(\sigma, \tau)|+|\widehat{Y(s, J)}(\sigma, \tau)|) d s \\
& \leq \alpha \lambda_{\sigma}^{p} \lambda_{\tau}^{p}+K \rho(1+|\widehat{J}(\sigma, \tau)|) t \\
& \leq \alpha \lambda_{\sigma}^{p} \lambda_{\tau}^{p}+\left(1+\alpha \lambda_{\sigma}^{p} \lambda_{\tau}^{p}\right) \sum_{i=1}^{1} \frac{\rho^{i} t^{i} K^{i}}{i !},
\end{aligned}
$$

where $\sigma, \tau \in \Gamma$ and $t \in[0, L]$.

Then we assume that, for some $n \geq 1$ and $t \in[0, L], T_{n}(t)$ has been defined and has the properties shown above. Then, by Theorem 18 , we find that $T_{n}(\cdot)$ is continuous; thus

$$
Y\left(\cdot, T_{n}(\cdot)\right):[0, L] \longrightarrow \mathbb{C}
$$

is also continuous, which means that the function $\left.Y \widehat{\left(s, T_{n}(s)\right.}\right)(\sigma, \tau) \widehat{N(s)}(\sigma, \tau), s \in[0, L]$, is measurable. On the other hand, we have

$$
\left.\mid Y \widehat{\left(s, T_{n}(s)\right.}\right)(\sigma, \tau) \widehat{N(s)}(\sigma, \tau) \mid \leq \alpha_{1} \lambda_{\sigma}^{p} \lambda_{\tau}^{p}
$$

$$
\sigma, \tau, \in \Gamma, s \in[0, L],
$$

where $\alpha_{1}=K \rho\left(1+\mathrm{e}^{\rho L K}+\alpha+\alpha \mathrm{e}^{\rho L K}\right)$. By Theorem 16, for any $t \epsilon$ $[0, L]$, the integral $\int_{0}^{t} Y\left(s, T_{n}(s)\right) * N(s) d s$ exists and belongs to $\mathscr{L}$. Similarly, for any $t \in[0, L]$, the integral $\int_{0}^{t} X\left(s, T_{n}(s)\right) d s$ also exists and belongs to $\mathscr{L}$.

Now we define a map $T_{n+1}:[0, L] \rightarrow \mathscr{L}$ as follows:

$$
\begin{aligned}
T_{n+1}(t)=J & +\int_{0}^{t} X\left(s, T_{n}(s)\right) d s \\
& +\int_{0}^{t} Y\left(s, T_{n}(s)\right) * N(s) d s, \quad t \in[0, L] .
\end{aligned}
$$

Obviously, for any $\sigma, \tau \in \Gamma$, the function $\widehat{T_{n+1}(\cdot)}(\sigma, \tau):[0$, $L] \rightarrow \mathbb{C}$ is continuous. Furthermore, because $X$ and $Y$ satisfy a locally uniform Lipschitz condition and locally uniform linear growth condition, we have

$$
\begin{aligned}
& \left.\left|\widehat{T_{n+1}(t)}(\sigma, \tau)\right| \leq \widehat{J}(\sigma, \tau)+\int_{0}^{t} \mid X \widehat{\left(s, T_{n}(s)\right.}\right)(\sigma, \tau) \mid d s \\
& \left.\quad+\int_{0}^{t} \mid Y \widehat{\left(s, T_{n}(s)\right.}\right)(\sigma, \tau)|| \widehat{N(s)}(\sigma, \tau) \mid d s \\
& \quad \leq \alpha \lambda_{\sigma}^{p} \lambda_{\tau}^{p}+K \int_{0}^{t}\left(\mid X \widehat{\left(s, T_{n}(s)\right.}\right)(\sigma, \tau) \mid \\
& \left.\left.\quad+\mid Y \widehat{\left(s, T_{n}(s)\right.}\right)(\sigma, \tau) \mid\right) d s \leq \alpha \lambda_{\sigma}^{p} \lambda_{\tau}^{p}
\end{aligned}
$$

$$
\begin{aligned}
& +K \rho \int_{0}^{t}\left(1+\left|\widehat{T_{n}(s)}(\sigma, \tau)\right|\right) d s \leq \alpha \lambda_{\sigma}^{p} \lambda_{\tau}^{p}+K \rho t \\
& +K \rho \int_{0}^{t}\left|\widehat{T_{n}(s)}(\sigma, \tau)\right| d s .
\end{aligned}
$$

Note that

$$
\begin{aligned}
\int_{0}^{t}\left|\widehat{T_{n}(s)}(\sigma, \tau)\right| d s \leq & \alpha t \lambda_{\sigma}^{p} \lambda_{\tau}^{p} \\
& +\left(1+\alpha \lambda_{\sigma}^{p} \lambda_{\tau}^{p}\right) \sum_{i=1}^{n} \frac{\rho^{i} K^{i} t^{(i+1)}}{(i+1) !}
\end{aligned}
$$

So we obtain that, for any $\sigma, \tau \in \Gamma$,

$$
\begin{aligned}
& \int_{0}^{t}\left|\widehat{T_{n+1}(s)}(\sigma, \tau)\right| d s \\
& \leq \alpha \lambda_{\sigma}^{p} \lambda_{\tau}^{p}+K \rho t \\
& \quad+K \rho\left[\alpha t \lambda_{\sigma}^{p} \lambda_{\tau}^{p}+\left(1+\alpha \lambda_{\sigma}^{p} \lambda_{\tau}^{p}\right) \sum_{i=1}^{n} \frac{\rho^{i} K^{i} t^{(i+1)}}{(i+1) !}\right] \\
& \quad \leq \alpha \lambda_{\sigma}^{p} \lambda_{\tau}^{p}+\left(1+\alpha \lambda_{\sigma}^{p} \lambda_{\tau}^{p}\right) \sum_{i=1}^{n+1} \frac{\rho^{i} K^{i} t^{i}}{i !} .
\end{aligned}
$$

Therefore, by induction, we come to our result.

Next, we show that, for any $t \in[0, L],\left(T_{n}(t)\right)_{n \geq 1}$ converges in $\mathscr{L}$. Indeed, by induction, for any $\sigma, \tau \in \Gamma$ and $t \in[0, L]$, we have

$$
\left|\widehat{T_{n}(t)}(\sigma, \tau)-\widehat{T_{n-1}(t)}(\sigma, \tau)\right| \leq\left(1+\alpha \lambda_{\sigma}^{p} \lambda_{\tau}^{p}\right) \frac{\rho^{n} K^{n} t^{n}}{n !} .
$$

So, for any $t \in[0, L]$ and $\sigma, \tau \in \Gamma$, the sequence $\left(\widehat{T_{n}(t)}(\sigma\right.$, $\tau))_{n \geq 1}$ converges in $\mathscr{L}$. On the other hand, from the properties of $T_{n}(t)$, we have

$$
\left|\widehat{T_{n}(t)}(\sigma, \tau)\right| \leq \alpha_{2} \lambda_{\sigma}^{p} \lambda_{\tau}^{p}
$$

where $\alpha_{2}=\alpha+\mathrm{e}^{\rho K t}+\alpha \mathrm{e}^{\rho K t}$. Thus, it follows from Theorem 14 that for any $t \in[0, L],\left(T_{n}(t)\right)_{n \geq 1}$ converges in $\mathscr{L}$.

We let

$$
T(t)=\lim _{n \rightarrow \infty} T_{n}(t), \quad t \in[0, L]
$$

Then, it is easy to see that

$$
|\widehat{T(t)}(\sigma, \tau)| \leq \alpha_{2} \lambda_{\sigma}^{p} \lambda_{\tau}^{p}
$$

Moreover, for $t \in[0, L], n, m \geq 1$, and $\sigma, \tau \in \Gamma$, we have

$$
\begin{aligned}
& \left|\widehat{T_{n+m}(t)}(\sigma, \tau)-\widehat{T_{n}(t)}(\sigma, \tau)\right| \\
& \quad \leq \sum_{i=n+1}^{n+m}\left|\widehat{T_{i}(t)}(\sigma, \tau)-\widehat{T_{i-1}(t)}(\sigma, \tau)\right| \\
& \quad \leq\left(1+\alpha \lambda_{\sigma}^{p} \lambda_{\tau}^{p}\right) \sum_{i=n+1}^{\infty} \frac{\rho^{i} K^{i} t^{i}}{i !} .
\end{aligned}
$$


So

$$
\begin{array}{r}
\sup _{0 \leq t \leq L}\left|\widehat{T(t)}(\sigma, \tau)-\widehat{T_{n}(t)}(\sigma, \tau)\right| \\
\quad \leq\left(1+\alpha \lambda_{\sigma}^{p} \lambda_{\tau}^{p}\right) \sum_{i=n+1}^{\infty} \frac{\rho^{i} K^{i} L^{i}}{i !},
\end{array}
$$

which means that the function $\widehat{T(\cdot)}(\sigma, \tau):[0, L] \rightarrow \mathbb{C}$ is continuous. Then, by Theorem 18 , the function $T(\cdot):[0, L] \rightarrow$ $\mathscr{L}$ is continuous.

In the same manner as above, we can show that, for each $t \in[0, L]$, the integrals $\int_{0}^{t} X(s, T(s)) d s$ and $\int_{0}^{t} Y(s, T(s)) *$ $N(s) d s$ exist and belong to $\mathscr{L}$. Further, by the dominated convergence theorem, we find that, for any $t \in[0, L]$ and $\sigma, \tau \in \Gamma$

$$
\begin{gathered}
\left.\left.\int_{0}^{t} X \widehat{\left(s, T_{n}(s)\right.}\right)(\sigma, \tau) d s \longrightarrow \int_{0}^{t} X \widehat{(s, T(s)}\right)(\sigma, \tau) d s, \\
\left.\int_{0}^{t} Y \widehat{\left(s, T_{n}(s)\right.}\right)(\sigma, \tau) \widehat{N(s)}(\sigma, \tau) d s \longrightarrow \\
\left.\int_{0}^{t} Y \widehat{(s, T(s)}\right)(\sigma, \tau) \widehat{N(s)}(\sigma, \tau) d s .
\end{gathered}
$$

Note that, for any $t \in[0, L]$ and $\sigma, \tau \in \Gamma$, we have

$$
\begin{aligned}
\widehat{T_{n+1}(t)} & (\sigma, \tau) \\
= & \left.\widehat{J}(\sigma, \tau)+\int_{0}^{t} X \widehat{\left(s, T_{n}(s)\right.}\right)(\sigma, \tau) d s \\
& \left.+\int_{0}^{t} Y \widehat{\left(s, T_{n}(s)\right.}\right)(\sigma, \tau) \widehat{N(s)}(\sigma, \tau) d s .
\end{aligned}
$$

Thus, by taking limit, we get

$$
\begin{aligned}
\widehat{T(t)}(\sigma, \tau)= & \left.\widehat{J}(\sigma, \tau)+\int_{0}^{t} X \widehat{(s, T(s)}\right)(\sigma, \tau) d s \\
& \left.+\int_{0}^{t} Y \widehat{(s, T(s)}\right)(\sigma, \tau) \widehat{N(s)}(\sigma, \tau) d s .
\end{aligned}
$$

which means that, for any $t \in[0, L]$, we have

$$
\begin{aligned}
T(t)= & J+\int_{0}^{t} X(s, T(s)) d s \\
& +\int_{0}^{t} Y(s, T(s)) * N(s) d s .
\end{aligned}
$$

So the map $T:[0, L] \rightarrow \mathscr{L}$ is a continuous solution to (35).

Finally, we prove the uniqueness. Assume that $S:[0$, $L] \rightarrow \mathscr{L}$ is another continuous solution to (35). Then together with $X$ and $Y$ satisfying a locally uniform Lipschitz condition and locally uniform linear growth condition, for each $t \in$ $[0, L]$ and $\sigma, \tau \in \Gamma$, we have

$$
\begin{aligned}
& |\widehat{T(t)}(\sigma, \tau)-\widehat{S(t)}(\sigma, \tau)| \\
& \left.\left.\quad \leq \int_{0}^{t} \mid X \widehat{(s, T(s)}\right)(\sigma, \tau)-X \widehat{(s, S(s)}\right)(\sigma, \tau) \mid d s
\end{aligned}
$$

$$
\begin{aligned}
& \left.\left.+\int_{0}^{t} \mid Y \widehat{(s, T(s)}\right)(\sigma, \tau)-Y \widehat{(s, S(s)}\right)(\sigma, \tau) \mid \\
& \cdot|\widehat{N(s)}(\sigma, \tau)| d s \\
& \leq \rho K \int_{0}^{t}|\widehat{T(s)}(\sigma, \tau)-\widehat{S(s)}(\sigma, \tau)| d s .
\end{aligned}
$$

By Gronwall inequality, we obtain that

$$
|\widehat{T(t)}(\sigma, \tau)-\widehat{S(t)}(\sigma, \tau)|=0, \quad t \in[0, L] .
$$

By Lemma $10, T(t)=S(t), t \in[0, L]$; that is, the solution is unique.

In the following, we always assume that the map $X, Y$ : $\mathbb{R}_{+} \times \mathscr{L} \rightarrow \mathscr{L}$ and the noise process $(N(t))_{t \in \mathbb{R}_{+}}$are the same as in Theorem 20. For $J \in \mathscr{L}$, we denote by $\left\{T(t, J) \mid t \in \mathbb{R}_{+}\right\}$ the solutions of (1) with $J$ being its initial value. The following theorem shows that the solution to (1) continuously depends on the initial value.

Theorem 21. Let $J_{1}, J_{2} \in \mathscr{L}$ be given. Then, for any $0 \leq L<$ $+\infty$, there exists constant $\rho \geq 0$, such that, for any $\sigma, \tau \in \Gamma$ and $t, s \in[0, L]$, one has

$$
\begin{aligned}
& \left.\mid \widehat{T\left(t, J_{1}\right.}\right)(\sigma, \tau)-\widehat{T\left(s, J_{2}\right)}(\sigma, \tau) \mid \\
& \quad \leq \mathrm{e}^{\rho K L}\left[\left|\widehat{J_{1}}(\sigma, \tau)-\widehat{J_{2}}(\sigma, \tau)\right|\right. \\
& \left.\quad+|t-s|\left(1+\left|\widehat{J_{1}}(\sigma, \tau)\right|+\left|\widehat{J_{2}}(\sigma, \tau)\right|\right)\right] .
\end{aligned}
$$

Proof. For $0 \leq L<+\infty$, let $p \geq 0, \rho>0$, and $K \geq 1$ be such that, for all $t \in[0, L]$ and $\sigma, \tau \in \Gamma$, we have

(1) $|\widehat{N(t)}(\sigma, \tau)| \leq K$;

(2) $|\widehat{X(t, A)}(\sigma, \tau)-\widehat{X(t, B)}(\sigma, \tau)|+\mid \widehat{Y(t, A)}(\sigma, \tau)-$ $\widehat{Y(t, B)}(\sigma, \tau)|\leq \rho| \widehat{A}(\sigma, \tau)-\widehat{B}(\sigma, \tau) \mid, A, B \in \mathscr{L} ;$

(3) $|\widehat{X(t, A)}(\sigma, \tau)|+|\widehat{X(t, B)}(\sigma, \tau)| \leq \rho(1+|\widehat{A}(\sigma, \tau)|)$.

It is easy to see that, for any $A \in \mathscr{L}$,

$$
\begin{aligned}
\widehat{T(t, A})(\sigma, \tau) \leq & |\widehat{A}(\sigma, \tau)| \\
& +\rho K \int_{0}^{t}(1+|\widehat{T(s, A)}(\sigma, \tau)|) d s,
\end{aligned}
$$

which implies that

$$
1+|\widehat{T(t, A)}(\sigma, \tau)| \leq(1+|\widehat{A}(\sigma, \tau)|) \mathrm{e}^{\rho K L} .
$$

Similarly, we can prove that

$$
\begin{aligned}
& \left.\mid \widehat{T\left(t, J_{1}\right.}\right)(\sigma, \tau)-\widehat{T\left(t, J_{2}\right)}(\sigma, \tau) \mid \\
& \quad \leq\left|\widehat{J_{1}}(\sigma, \tau)-\widehat{J_{2}}(\sigma, \tau)\right| \mathrm{e}^{\rho K L} .
\end{aligned}
$$


Then, using estimates (62) and (63), we have

$$
\begin{aligned}
& \left.\left|\widehat{T\left(t, J_{1}\right)}(\sigma, \tau)-\widehat{T\left(s, J_{2}\right)}(\sigma, \tau)\right| \leq \mid \widehat{T\left(t, J_{1}\right.}\right)(\sigma, \tau) \\
& -\widehat{T\left(t, J_{2}\right)}(\sigma, \tau)|+| \widehat{T\left(t, J_{2}\right)}(\sigma, \tau) \\
& \left.\quad-\widehat{T\left(s, J_{2}\right.}\right)(\sigma, \tau) \mid \leq \mathrm{e}^{\rho K L}\left[\left|\widehat{J_{1}}(\sigma, \tau)-\widehat{J_{2}}(\sigma, \tau)\right|\right. \\
& \left.\quad+|t-s|\left(1+\left|\widehat{J_{1}}(\sigma, \tau)\right|+\left|\widehat{J_{2}}(\sigma, \tau)\right|\right)\right] .
\end{aligned}
$$

\section{Conflicts of Interest}

The author declares that there are no conflicts of interest.

\section{Acknowledgments}

This work is supported by the National Natural Science Foundation of China (Grants nos. 11461061 and 11761047).

\section{References}

[1] T. Hida, Analysis of Brownian Functionals, Carleton Math, Carleton Univ., Ottawa, Canada, 1975.

[2] T. Hida, H.-H. Kuo, J. Potthoff, and L. Streit, White Noise: An Infinite Dimensional Calculus, Kluwer Academic, Dordrecht, Netherlands, 1993.

[3] Z. Y. Huang and J. A. Yan, Introduction to Infinite Dimensional Stochastic Analysis, Kluwer Academic, Dordrecht, Netherlands, 1999.

[4] N. Obata, "An analytic characterization of symbols of operators on white noise functionals," Journal of the Mathematical Society of Japan, vol. 45, no. 3, pp. 421-445, 1993.

[5] H. Holden, B. Oksendal, J. Uboe, and T. Zhang, Stochastic Partial Differential Equations, A Modeling, White Noise Functional Approach, Birkhauser, Boston, Mass, USA, 1996.

[6] H. H. Kuo, White Noise Distribution Theory, CRC Press, Boca Raton, Fla, USA, 1996.

[7] G. Da Prato and J. Zabczyk, Stochastic Equations in Infinite Dimensions, vol. 44, Cambridge University Press, Cambridge, UK, 1992.

[8] Y. Ito, "Generalized Poisson functionals," Probability Theory and Related Fields, vol. 77, no. 1, pp. 1-28, 1988.

[9] S. Albeverio, Y. L. Daletsky, Y. G. Kondratiev, and L. Streit, "Non-Gaussian infinite-dimensional analysis," Journal of Functional Analysis, vol. 138, no. 2, pp. 311-350, 1996.

[10] A. Barhoumi, H. Ouerdiane, and A. Riahi, "Pascal white noise calculus," Stochastics. An International Journal of Probability and Stochastic Processes, vol. 81, no. 3-4, pp. 323-343, 2009.

[11] G. Di Nunno, B. Oksendal, and F. Proske, "White noise analysis for Lévy processes," Journal of Functional Analysis, vol. 206, no. 1, pp. 109-148, 2004.

[12] Y.-J. Lee and H.-H. Shih, "The Segal-Bargmann transform for Lévy functionals," Journal of Functional Analysis, vol. 168, no. 1, pp. 46-83, 1999.

[13] N. Privault, "Stochastic analysis of Bernoulli processes," Probability Surveys, vol. 5, pp. 435-483, 2008.
[14] R. Motwani and P. Raghavan, Randomized Algorithms, Cambridge University Press, New York, NY, USA, 1995.

[15] J. Rudnick and G. Gaspari, Elements of the Random Walk, Cambridge University Press, Cambridge, UK, 2004.

[16] C. Wang and J. Chen, "Characterization theorems for generalized functionals of discrete-time normal martingale," Journal of Function Spaces, vol. 2015, Article ID 714745, 2015.

[17] C. Wang and J. Chen, "A characterization of operators on functionals of discrete-time normal martingales," Stochastic Analysis and Applications, vol. 35, no. 2, pp. 305-316, 2017.

[18] M. Émery, "A discrete approach to the chaotic representation property," in Séminaire de Probabilités XXXV, vol. 1755 of Lecture Notes in Mathematics, pp. 123-138, Springer, Berlin, Germany, 2001.

[19] C. Wang, Y. Lu, and H. Chai, "An alternative approach to Privault's discrete-time chaotic calculus," Journal of Mathematical Analysis and Applications, vol. 373, no. 2, pp. 643-654, 2011.

[20] C. Wang and J. Zhang, "Wick analysis for bernoulli noise functionals," Journal of Function Spaces, vol. 2014, Article ID 727341, 2014.

[21] I. M. Gel'fand and N. Y. Vilenkin, Generalized Functions, vol. 4, Academic Press, New York, NY, USA, 1964. 


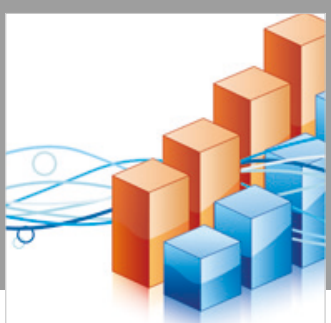

Advances in

Operations Research

\section{-n-m}
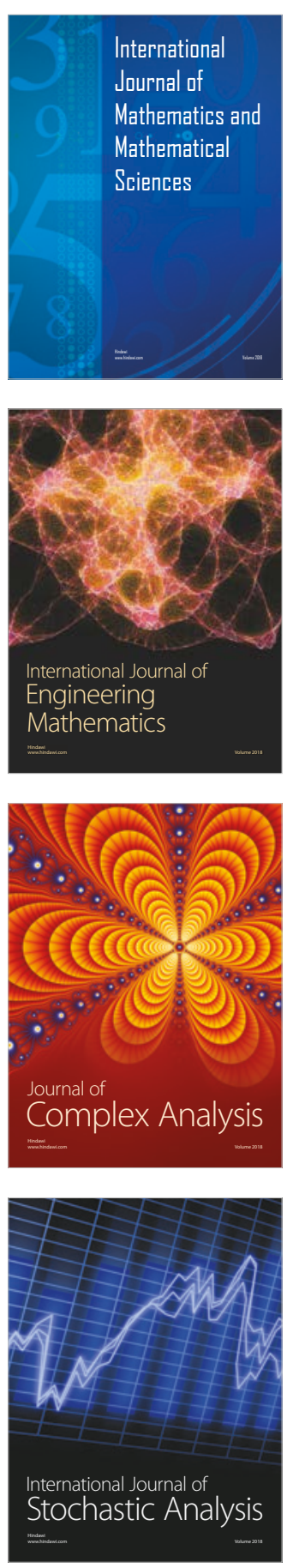
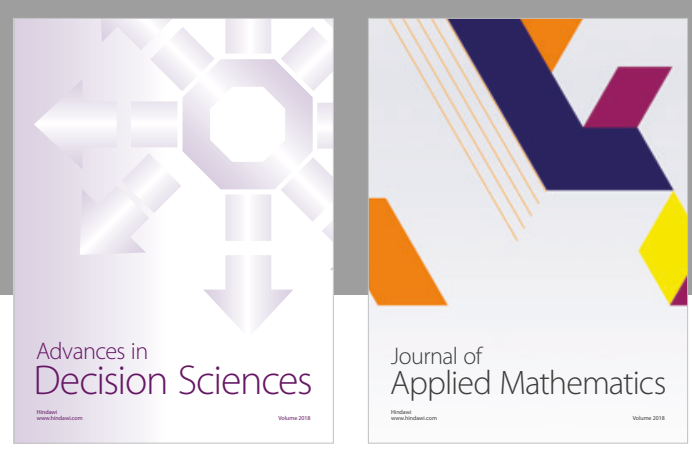

Journal of

Applied Mathematics
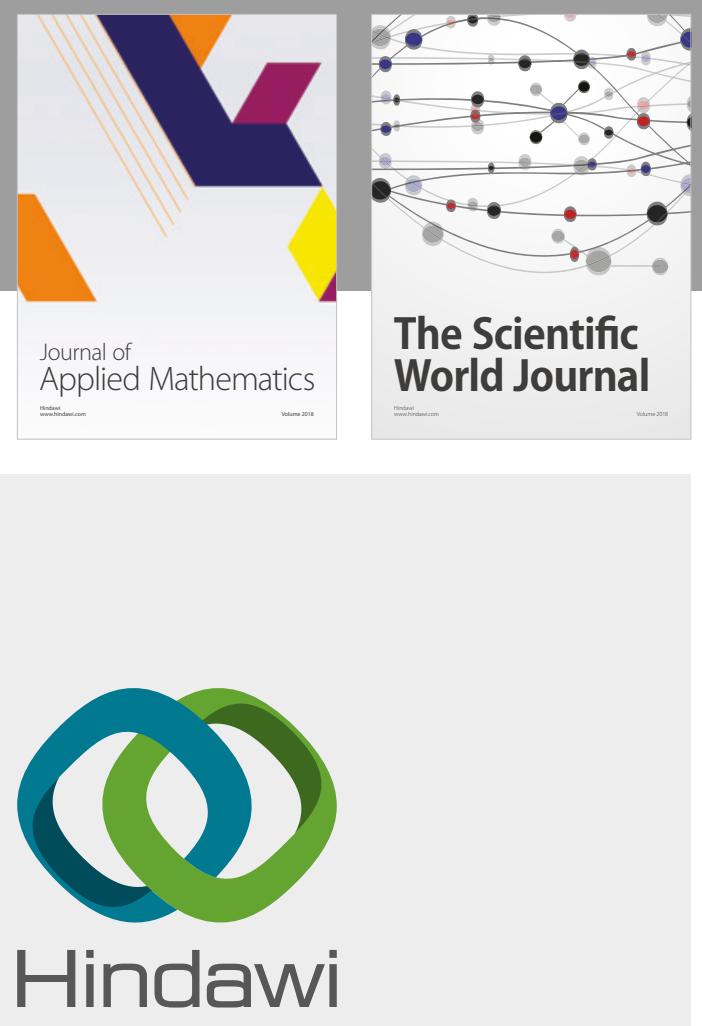

Submit your manuscripts at

www.hindawi.com

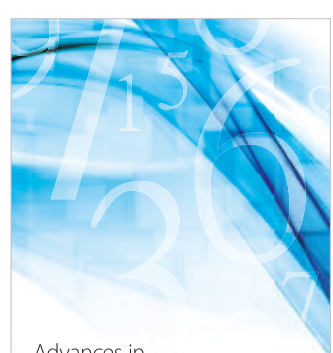

Advances in
Numerical Analysis
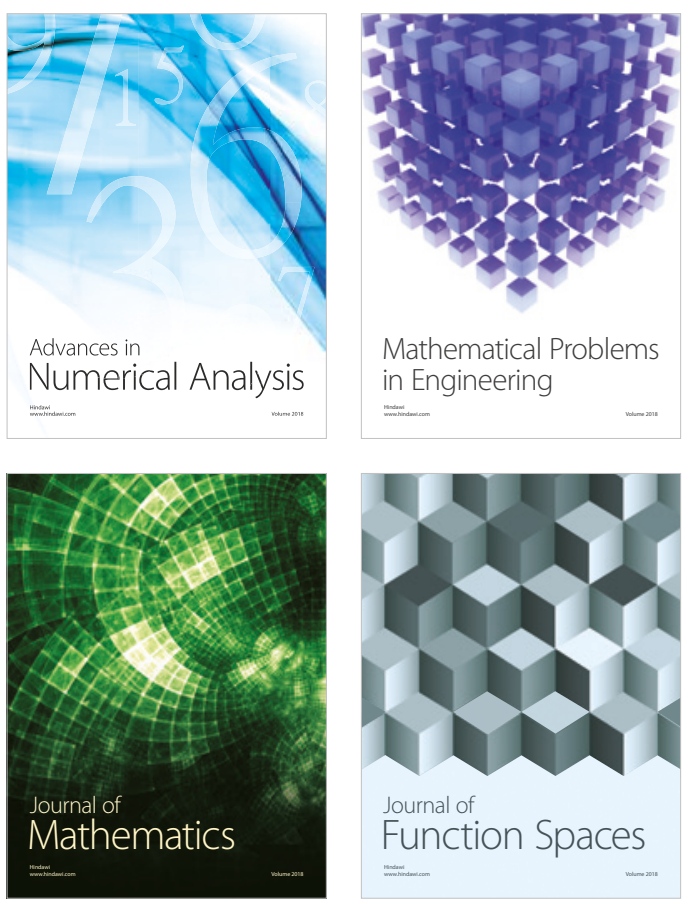

Mathematical Problems in Engineering

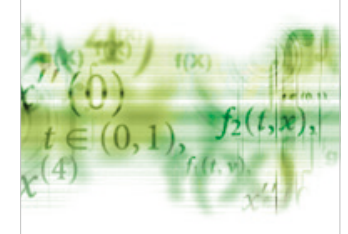

International Journal of

Differential Equations

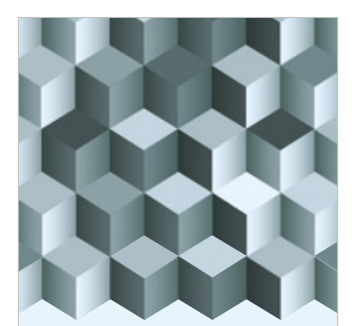

Journal of

Function Spaces

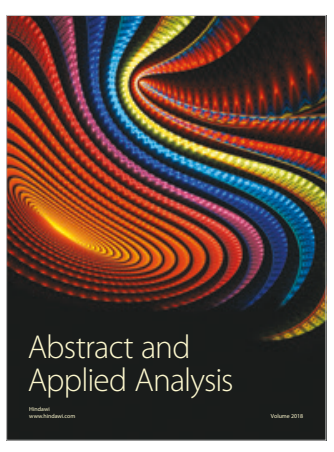

The Scientific

World Journal

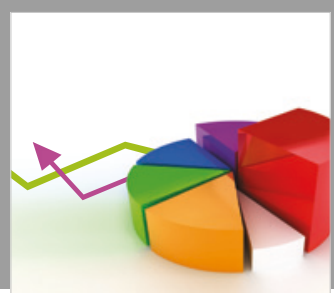

Journal of

Probability and Statistics
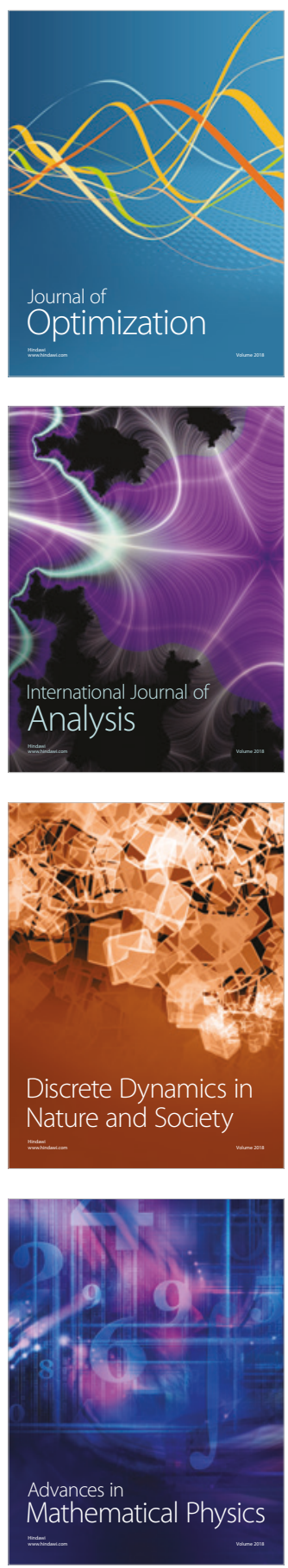